\title{
Serotonin Syndrome Associated with Antidepressant Discontinuation in a Neonate Following Drug Exposure During Pregnancy
}

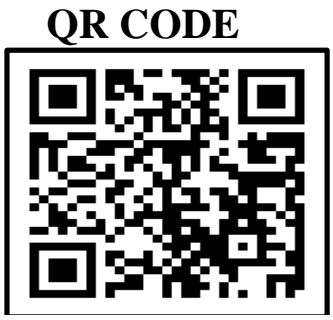

\section{DOLMA GURUNG ${ }^{1}$, SUNIL BORAH ${ }^{2}$, SAMEER WANGU*}

Sudden withdrawal of some drugs leads to withdrawal issues. We describe a baby girl who was indirectly exposed to venlafaxine as her mother was administered this drug during pregnancy for treatment of depression. The subject presented with involuntary movement of the limbs. The mother had also encountered similar scenario along with some other symptoms after she suddenly discontinued her medication. The symptoms of the mother completely resolved a few hours after the she took another dose of venlafaxine. The baby was kept under observation and recovered completely without any intervention. We suggest that healthcare professionals should be aware that patients may require tapering of anti-depressants before discontinuation.

KEYWORDS: Serotonin Syndrome, Withdrawal Symptoms, Antidepressant

\section{INTRODUCTION}

Abrupt discontinuation of many pharmaceuticals is associated with withdrawal symptoms. Antidepressant drugs are associated with a group of common symptoms after discontinuation. ${ }^{1}$ The first cases of antidepressant discontinuation syndrome were reported for imipramine by Andersen and Christiansen in 1959. Since then, this phenomenon has been described for different families of antidepressants: Tricyclics, Monoamine Oxidase Inhibitors, and more recently, selective serotonin reuptake inhibitors. Some newborns exposed during pregnancy to serotonin and norepinephrine reuptake inhibitors or selective serotonin reuptake inhibitors may present, at birth, symptoms such as respiratory distress, cyanosis, apnea, seizures, feeding difficulties, vomiting, hypoglycemia, muscle tone changes, tremors, or irritability. This is related to the direct toxic effect of selective serotonin reuptake inhibitors and serotonin and norepinephrine reuptake inhibitors and is known as antidepressant discontinuation syndrome or serotonin syndrome. There are very few documented and published cases in the literature on newborns exposed during pregnancy to selective serotonin reuptake inhibitors with subsequent development of serotonin syndrome.

\section{CASE REPORT}

We present the case of a 5-day old female baby who was referred from a rural civil hospital to our clinic for presenting involuntary movements of the four limbs from 2 days, lasting less than a minute. Birth weight of the baby was 3.5 kilograms. As per the medical history, only the mother had been taking venlafaxine treatment during pregnancy. There were no other perinatal incidents. Upon arrival at our centre, she was monitored with normal vital signs, and brain function monitoring was started by means of amplitudeintegrated electroencephalography, showing a continuous trace. Subsequently, a conventional electroencephalogram was performed, coinciding with frequent movements of the patient, which was reported as normal, without pathological graph elements of epileptiform morphology. Brain ultrasound showed no relevant alterations.

Toxicity determination was performed in urine, with negative result. Infectious, neurometabolic, and hormonal examinations were performed, without evidence of any pathological findings. The patient had normal results at physical and neurological examination at all times. The mother had also encountered dizziness, lightheadedness, excessive sweating, irritability, dysphoria, and insomnia and started performing similar movements few days after stopping the treatment with venlafaxine that she had received during pregnancy, so it was suspected that the condition was due to a serotonin syndrome caused by venlafaxine withdrawal. The mother also revealed that she fully recovered from her withdrawal symptoms few hours after she was given a dose of venlafaxine by her family physician and now, she continues with her

(C) Dolma Gurung et al. This is an open access article distributed under the terms of the Creative Commons Attribution License CC-BY-NC 4.o, which permits unrestricted use, distribution and reproduction in any medium, provided the use is not commercial and the original author(s) and source are cited. Submitted on: o6-Aug-2021; Accepted on: 26-Aug-2021 
prescription. The baby was kept under observation in our special care unit and no intervention was started. Her involuntary movements persisted intermittently, becoming less and less frequent, giving way completely after one week of life. The patient was discharged without incident and remained asymptomatic at weekly and monthly follow up.

\section{DISCUSSION}

The phenomena of discontinuation of serotonin reuptake inhibitors have been described since first half of zoth century, their appearance being unusual in the neonatal setting. It is well recognized that babies exposed to antidepressants in utero may develop symptoms of drug withdrawal following birth. Such symptoms include diarrhea, vomiting, hypothermia, restlessness, irritability, agitation, inadequate feeding, insomnia, hypoglycemia, respiratory distress, altered muscle tone, hyper-reflexia, tremors and seizures.

A urine toxicology screen when taken shortly after birth in symptomatic subjects can be used to exclude exposure to certain drugs. Venlafaxine, with noradrenergic and serotonergic action, is a relatively new antidepressant. However, in a clinical trial, Farah and Lauer described discontinuation episodes in some patients. ${ }^{2}$ Fava et al. found discontinuation symptoms in $78 \%$ of patients treated with sustained-release venlafaxine versus $28 \%$ in patients treated with placebo. ${ }^{3}$ The diagnosis was made after the exclusion of other causes of paroxysmal movements and confirmed when the same symptoms were observed in the mother after the drug was discontinued. Current evidence indicates that fetal exposure to selective serotonin reuptake inhibitors during the last trimester of pregnancy may lead to a neonatal behavioral syndrome, generally self-limiting. ${ }^{4}$ The decision to expose the fetus to antidepressant medications during pregnancy must be weighed against the risks of untreated maternal depression for both the mother and the fetus, so it should be used only if the potential benefits justify the risks to the fetus. Exposed newborns should be monitored after delivery for the direct toxic effects of this drug, drug discontinuation syndrome, and serotonin syndrome.

\section{CONCLUSION}

Discontinuation related adverse reactions are fairly common among antidepressant treated patients. This may also affect the baby in a similar way if these drugs are administered to the mother during pregnancy and abruptly stopped. Healthcare professionals should consider tapering the antidepressant dose gradually.

\section{REFERENCES}

1. Zajecka J, Tracy KA, Mitchell S. Discontinuation symptoms after treatment with serotonin reuptake inhibitors: a literature review. J Clin Psychiatry. 1997 Jul;58(7):291-7. https://doi.org/10.4088/jcp.v58no702

2. Farah A, Lauer TE. Possible venlafaxine withdrawal syndrome. Am J Psychiatry. 1996;153(4):576. https://doi.org/10.1176/ajp.153.4.576a

3. Fava M, Mulroy R, Alpert J, Nierenberg AA, Rosenbaum JF. Emergence of adverse events following discontinuation of treatment with extended-release venlafaxine. Am J Psychiatry. 1997;154(12):176o-2. https://doi.org/10.1176/ajp.154.12.176o

4. Moses-Kolko EL, Bogen D, Perel J, Bregar A, Uhl K et al. Neonatal signs after late in utero exposure to serotonin reuptake inhibitors: literature review and implications for clinical applications. JAMA. 2005 May 18;293(19):2372-83.

https://doi.org/10.1001/jama.293.19.2372

Cite this article as:

Gurung D, Borah S, Wangu S. Serotonin Syndrome Associated with Antidepressant Discontinuation in a Neonate Following Drug Exposure During Pregnancy. Int Healthc Res J. 2021;5(5):CR1-CR2. https://doi.org/10.26440/IHRJ/0505.08450

AUTHOR AFFILIATIONS: ( ${ }^{*}$ Corresponding Author)

1. MD (Obstetrics \& Gynaecology), Senior Consultant, Sikkim, India

2. MD (Paediatrics), Associate Consultant, Bhatinda, Punjab, India

3. MD (Paediatrics), Associate Consultant, Indore, India 